Europhysics Letters

PREPRINT

\title{
Scaling of the magnetic linear response in phase-ordering kinetics
}

\author{
Malte Henkel $^{1}$, Matthias Paessens ${ }^{1,2}$, and Michel Pleimling ${ }^{3}$ \\ 1 Laboratoire de Physique des Matériaux, (*) Université Henri Poincaré Nancy I, B.P. \\ 239, F-54506 Vandouvre lès Nancy Cedex, France \\ 2 Institut für Festkörperforschung (Theorie II), Forschungszentrum Jülich, D - 52425 \\ Jülich, Germany \\ 3 Institut für Theoretische Physik I, Universität Erlangen-Nürnberg, D - 91058 Erlan- \\ gen, Germany
}

PACS. 05.70.Ln - Nonequilibrium and irreversible thermodynamics.

PACS. 75.40.Gb - Dynamic properties.

PACS. 64.60.Ht - Dynamic critical phenomena.

\begin{abstract}
The scaling of the thermoremanent magnetization and of the dissipative part of the non-equilibrium magnetic susceptibility is analysed as a function of the waiting-time $s$ for a simple ferromagnet undergoing phase-ordering kinetics after a quench into the ferromagnetically ordered phase. Their scaling forms describe the cross-over between two power-law regimes governed by the non-equilibrium exponents $a$ and $\lambda_{R} / z$, respectively. A relation between $a$, the dynamical exponent $z$ and the equilibrium exponent $\eta$ is derived from scaling arguments. Explicit tests in the Glauber-Ising model and the kinetic spherical model are presented.
\end{abstract}

The study of non-equilibrium critical phenomena originated from studies of the dynamic behaviour of glassy systems, but has also produced new insights into the behaviour of the conceptually simpler ferromagnetic systems, see [1, 2, 3, 4, for reviews. Since the latter are considerably more tractable, it might be hoped that insights gained from studying the kinetics of simple ferromagnets could provide clues for the comprehension of the former. In this letter, we consider simple ferromagnetic spin systems, quenched from a disordered initial state to below its critical temperature $T_{c}>0$. We shall work with a non-conserved order parameter throughout.

Then the system undergoes phase ordering, that is domains of a time-dependent typical size $L(t) \sim t^{1 / z}$ form and grow, where $z$ is the dynamical exponent [2]. The resulting slow evolution of the system is more fully revealed through the study of two-time quantities, such as the two-time autocorrelation function $C(t, s)$ and the autoresponse function $R(t, s)$

$$
C(t, s)=\langle\phi(t) \phi(s)\rangle, R(t, s)=\left.\frac{\delta\langle\phi(t)\rangle}{\delta h(s)}\right|_{h=0}
$$

$\left({ }^{*}\right)$ Laboratoire associé au CNRS UMR 7556

(c) EDP Sciences 
where $\phi$ is the order parameter, $h$ the conjugate magnetic field, $t$ is called the observation time and $s$ the waiting time. Ageing occurs in the regime when $s$ and $\tau=t-s>0$ are

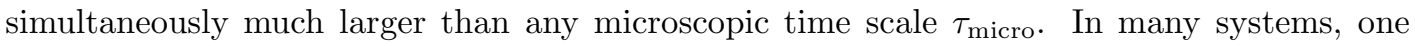
finds in the ageing regime a scaling behaviour, see [1] 3 ]

$$
C(t, s)=s^{-b} f_{C}(t / s), R(t, s)=s^{-1-a} f_{R}(t / s)
$$

where $a$ and $b$ are non-equilibrium exponents and the scaling functions behave for large arguments $x=t / s \gg 1$ asymptotically as

$$
f_{C}(x) \sim x^{-\lambda_{C} / z}, f_{R}(x) \sim x^{-\lambda_{R} / z}
$$

where $\lambda_{C}$ and $\lambda_{R}$ are the autocorrelation [5] 6] and autoresponse [7] exponents, respectively. For the usually studied case of an initial state without long-range correlations, it is generally accepted that $\lambda_{C}=\lambda_{R}=\lambda$. Combinations of rigorous results and of heuristic scaling arguments were used to derive the bounds $d / 2 \leq \lambda \lesssim d[5$, 8].

It has been proposed recently that one might be able to go beyond mere dynamical scale invariance as expressed by eq. (2) to a group of local scale transformations related to conformal transformations in time [9]. If that hypothesis applies, the form of the scaling function

$$
f_{R}(x)=r_{0} x^{1+a-\lambda_{R} / z}(x-1)^{-1-a}
$$

is completely fixed ( $r_{0}$ is a normalization constant). Eq. (44) has been confirmed in several models, especially, through extensive simulations, in the $2 D$ and $3 D$ Glauber-Ising models [10].

We are interested in the value of the non-equilibrium exponent $a$. In quite a few models, values of $a$ were obtained, see [3] for a review, but no clear picture has yet emerged. In the exactly solved $1 D$ Glauber-Ising model, one has $a=0$ 11, 12. However, the value of $a$ in the $2 D$ and $3 D$ kinetic Glauber-Ising models and the kinetic spherical models has been debated recently. In the $2 D$ and $3 D$ Glauber-Ising model, analytical [13] and numerical [10] results indicate $a=1 / 2$. In the exactly solvable spherical model, one reads off $a=d / 2-1$ for all spatial dimensions $d>2$ from the exact result for $R(t, s)$ [14, 15, 16, 18.

An alternative route has been followed by Corberi, Lippiello and Zannetti 1718 19. They studied the two-time correlation function $C(t, s)$ and the susceptibility function $\chi(t, s)$ in the $\mathrm{O}(n)$ vector model. Separating the two-time autocorrelation function $C(t, s)=C_{\mathrm{st}}(t-s)+$ $C_{\text {age }}(t / s)$ into a stationary part and an ageing part and similarly for the ZFC susceptibility $\chi(t, s)=\chi_{\mathrm{st}}(t-s)+\chi_{\text {age }}(t, s)$, where $\chi_{\mathrm{st}}$ is defined such that it satisfies the fluctuationdissipation theorem together with $C_{\text {eq }}$, they extract an exponent $\hat{a}$ from the scaling behaviour $\chi_{\text {age }}(t, s)=s^{-\hat{a}} \hat{\chi}(t / s)$ and claim that $\hat{a}=a$. This was backed up by the assertion that the scaling of $\chi_{\text {age }}(t, s)$ were anomalous [18. The same procedure was applied to simulational data of the ZFC susceptibility from the $2 D$ and $3 D$ Glauber-Ising model. If $a_{n}$ is the value of $\hat{a}$ in the $\mathrm{O}(n)$-model, they propose [17, 18, 19]

$$
a_{1}=\left\{\begin{array}{ll}
(d-1) / 4 & ; d<3 \\
1 / 2 & ; d>3
\end{array} \quad, a_{\infty}= \begin{cases}(d-2) / 2 & ; 2<d<4 \\
1 & ; d>4\end{cases}\right.
$$

for the Glauber-Ising $(n=1)$ and the spherical model $(n=\infty)$, respectively. However, since $a_{\infty} \neq d / 2-1=a$ for the spherical model, although even Corberi et al. do recover $a=d / 2-1$ from $R(t, s)$, see eq. (83) in [18, their claim of an anomalous scaling of $\chi(t, s)$ as raised in [18,19] appears to be ill-founded.

Our results in this letter are as follows: one should distinguish between those systems (called here class $S$ ) with a short-ranged (exponential) decay of the equilibrium connected 
spin-spin correlator in the ordered phase and those (called here class L) for which there remain long-range (algebraic) correlations in the ordered phase. The Glauber-Ising model in $d>1$ belongs to class $\mathrm{S}$ while the spherical model and the $2 D \mathrm{XY}$-model are members of class $\mathrm{L}[20$. Then

$$
a= \begin{cases}1 / z & ; \text { class S } \\ (d-2+\eta) / z & ; \text { class L }\end{cases}
$$

where $\eta$ is a well-known equilibrium critical exponent. Since for the Glauber-Ising model $z=2$ [2], one recovers the well-known $a=1 / 2$ for class $\mathrm{S}$ in all dimensions $d>1$. These results will be derived by comparing the scaling form of the dissipative part $\chi^{\prime \prime}$ of the nonequilibrium susceptibility (see eq. (11) below) with heuristic expectations which for class $\mathrm{S}$ amount to the generally accepted idea that the ageing comes from the movement of the domain walls which separate the ordered domains 1, 2, 13. In addition, we shall derive a more complete scaling form for the thermoremanent magnetization $M_{\mathrm{TRM}}(t, s)$ in the limit of large waiting times $s \gg 1$

$$
M_{\mathrm{TRM}}(t, s) / h=\int_{0}^{s} \mathrm{~d} u R(t, u)=s^{-a} f_{M}(t / s)+s^{-\lambda_{R} / z} g_{M}(t / s)
$$

provided the system was initially prepared at infinite temperature. Eq. (7) implies that the behaviour of $M_{\mathrm{TRM}}$ will be one of a cross-over between two distinct regimes [15, 19. In practice, the cross-over region may well be large. If local scale invariance 9 holds, the scaling functions can be found from eq. (4) and read explicitly ( $r_{0,1}$ are non-universal constants)

$$
f_{M}(x)=r_{0} x^{-\lambda_{R} / z}{ }_{2} F_{1}\left(1+a, \frac{\lambda_{R}}{z}-a ; \frac{\lambda_{R}}{z}-a+1 ; x^{-1}\right), g_{M}(x) \approx r_{1} x^{-\lambda_{R} / z}
$$

The new and explicit forms of these scaling functions allow for a considerably more precise test of the cross-over scaling of $M_{\mathrm{TRM}}$ than had been possible before. In particular, having fixed the parameters $r_{0,1}$ for a given value of $x$, a precise prediction for the scaling of $M_{\mathrm{TRM}}$ for any other value of $x$, without any free parameter, is obtained. We shall use this idea to perform a new type of precision test on the scaling of $M_{\mathrm{TRM}}(t, s)$ for class $\mathrm{S}$ systems and in particular test for the value of the exponent $a$.

We shall first derive the scaling forms of $\chi^{\prime \prime}$ and $M_{\text {TRM }}$ as a function of the waiting time $s$ and prove (6) by relating heuristic ideas on coarsening to scaling arguments. Then we shall describe numerical tests of (788) in the spherical and Glauber-Ising models.

Consider the time-dependent response of a ferromagnetic system to a sinusoidal magnetic field with frequency $\omega$. The imaginary part of the susceptibility is given by [1, 4]

$$
\chi^{\prime \prime}(\omega ; s)=\int_{0}^{s} \mathrm{~d} u R(s, u) \sin (\omega(s-u))
$$

which can be analysed using properties of the autoresponse function $R(s, u)$. Here, the time difference $\tau=s-u$ plays a central role and depending on its value an equilibrium or else an ageing behaviour is obtained. Specifically, it can be shown [15] that there is a time scale $t_{p} \sim s^{\zeta}$ such that $0<\zeta<1$ (explicitly, $\zeta=4 /(d+2)$ for the spherical model [15]) on which the breaking of the fluctuation-dissipation relation occurs and furthermore $R(t, s) \simeq R_{\mathrm{eq}}(t-s)$ for $t-s \lesssim t_{p}$ and $R(t, s)=R_{\text {scal }}(t, s)$ is given by (2) for $t_{p} \lesssim t-s$. For $u \approx s$ one measures the response with respect to a change in the initial conditions and $R \approx R_{\text {ini }}(t) \sim t^{-\lambda_{R} / z}$ [2]. Besides $t_{p}$, and following [15, 4, we therefore introduce a third time scale $t_{\varepsilon}$ such that $s-t_{\varepsilon}=\mathrm{O}(1)$. 
Changing variables and splitting the integral into three terms, one has

$$
\begin{aligned}
& \chi^{\prime \prime}(\omega ; s)=\int_{0}^{s} \mathrm{~d} \tau R(s, s-\tau) \sin \omega \tau \\
& =\int_{0}^{t_{p}} \mathrm{~d} \tau R(s, s-\tau) \sin \omega \tau+\int_{t_{p}}^{t_{\varepsilon}} \mathrm{d} \tau R(s, s-\tau) \sin \omega \tau+\int_{t_{\varepsilon}}^{s} \mathrm{~d} \tau R(s, s-\tau) \sin \omega \tau \\
& \simeq \int_{0}^{t_{p}} \mathrm{~d} \tau R_{\text {eq }}(\tau) \sin \omega \tau+s^{-a} \int_{t_{p} / s}^{t_{\varepsilon} / s} \mathrm{~d} v f_{R}\left(\frac{1}{1-v}\right) \frac{\sin (\omega s v)}{(1-v)^{1+a}}+s^{-\lambda_{R} / z} \int_{t_{\varepsilon}}^{s} \mathrm{~d} \tau c_{0} \sin \omega \tau
\end{aligned}
$$

where $c_{0}$ is a constant. In the third line, we used the asymptotic form of $R(s, u)$ in the three regimes. This is justified if for sufficiently large values of $s$, the cross-over between these regimes is rapid. In a given system, this can be checked by looking for a rapid cross-over in the fluctuation-dissipation ratio (see e.g. 1,3,4) between the initial equilibrium and the later ageing regime, see [16, 18. Now, in the limit $s \rightarrow \infty$ one has (i) $t_{p} \rightarrow \infty$, (ii) $t_{p} / s \rightarrow 0$ and (iii) $t_{\varepsilon} / s \rightarrow 1$. We then find

$$
\chi^{\prime \prime}(\omega ; s)=\chi_{1}(\omega)+s^{-a} \chi_{2}(\omega s)+\mathrm{O}\left(s^{-\lambda_{R} / z}\right)
$$

For systems of class $\mathrm{S}$, it is generally expected that the dynamics proceeds via domain growth, due to interface motion between well-ordered domains [1,2, 14, 13]. Therefore, the susceptibility $\chi$ should decompose into a stationary part $\chi$ eq to which the spins in the bulk of the domains contribute and an ageing part $\chi_{\text {ag }}$ which comes only from the domain walls, with a length scale given by the typical distance $L(s)$. The imaginary part of the susceptibility should read 1, 14,13

$$
\chi^{\prime \prime}(\omega ; s)=\chi_{\mathrm{eq}}^{\prime \prime}(\omega)+L(s)^{-1} \chi_{\mathrm{ag}}^{\prime \prime}(\omega s)
$$

Comparing with the general scaling form (11), we see that the first term corresponds to the equilibrium term in (12), the second to the ageing contribution and the third is a correction term. From the scaling $L(s) \sim s^{1 / z}$ of the domains one indeed recovers the first part of (6).

For systems of class $L$ the long-range fluctuations turn the ordered domains into fractal objects and we can no longer assume the existence of simple and well-separated domain walls. Rather, we anticipate instead of (12) the form

$$
\chi^{\prime \prime}(\omega ; s)=\chi_{\text {eq }}^{\prime \prime}(\omega)+L(s)^{-(d-2+\eta)} \chi_{\mathrm{ag}}^{\prime \prime}(\omega s)
$$

(up to possible logarithmic factors, which arise e.g. in the $2 D \mathrm{XY}$ model [21]). The ageing part should come from the moving fractal domain boundaries. The simplest way to describe the time-dependence of this is to assume that all length scales are measured in terms of the domain size $L(t)$. If that is so, the spin-spin correlator should read $C(\boldsymbol{r}, t) \approx C_{\mathrm{qs}}(\boldsymbol{r}, L(t)) \sim$ $r^{-(d-2+\eta)} c(r / L(t))$ in terms of a quasi-stationary correlator $C_{\mathrm{qs}}$ and where $c$ is some scaling function. The time-dependent contribution to the susceptibility per volume $V$ should then come from $\chi_{\mathrm{qs}} \sim V^{-1} \int_{V} \mathrm{~d} \boldsymbol{r} C_{\mathrm{qs}}(\boldsymbol{r}, L(t)) \sim L(t)^{-(d-2+\eta)}$ and this leads to the desired result (13). The rest of the analysis then goes through as before and the second part of (6) follows. Of course, we must assume that the scaling functions $\chi_{\text {ag }}^{\prime \prime}(x)$ have no singularity as $x \rightarrow \infty$.

Eq. (6) is confirmed for class L by all known results for simple ferromagnets. First, for the short-ranged spherical model either without [14,15, 16, 3] or else with [7] initial longrange correlations one has $z=2, \eta=0$. From the exact results for $R(t, s)$ one recovers indeed $a=d / 2-1$ as expected from (6). Second, for the spherical model with long-range interactions of the form $J(\boldsymbol{r}) \sim r^{-d-\sigma}$ and $0<\sigma<\min (d, 2)$ one has $z=\sigma, \eta=2-\sigma$ and $a=d / \sigma-1$ [22], 


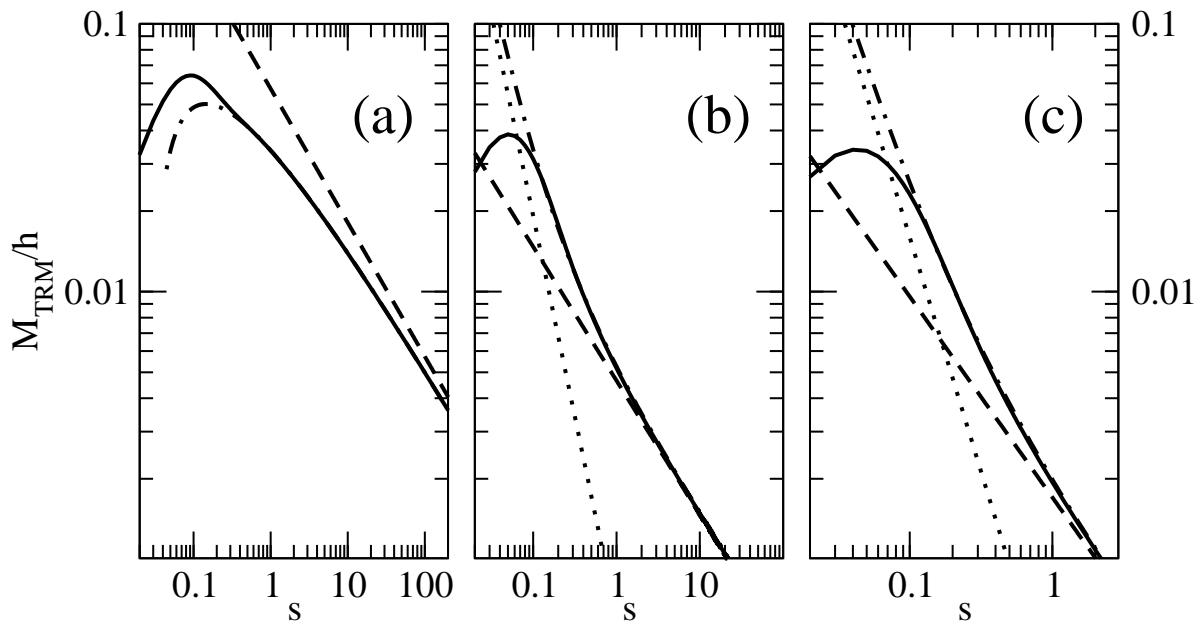

Fig. 1 - Scaling of the thermoremanent magnetization $M_{\mathrm{TRM}}(t, s)$ (full curve) at $T=2$ as a function of the waiting time $s$ in the spherical model for $x=t / s=5$. (a) $d=3$ and $S_{0}=0$, (b) $d=3$ and $S_{0}=0.5$, (c) $d=3.5$ and $S_{0}=0.5$. The dashed and dotted lines show the leading contributions $\sim s^{-a}$ and $\sim s^{-\lambda_{R} / z}$, respectively, whereas the dash-dotted lines give their sum.

as it should be. In these models, $b=0$ (see eq. (21)). Third, in the $2 D \mathrm{XY}$-model one has $z=2[2$ and $a=b=\eta(T) / 2$ for both fully ordered and fully disordered initial conditions as shown analytically and through simulations [21], where $\eta(T)$ depends on temperature. Fourth, the defining condition for class $\mathrm{L}$ is also satisfied for non-equilibrium critical dynamics. There, it is known that $a=b=2 \beta / \nu z=(d-2+\eta) / z$, see $[3$, in agreement with (6). Finally, for the $1 D$ Glauber-Ising model ageing occurs only at the critical point $T_{c}=0$. The known exponent $\eta=1$ yields $a=0$, as it should be $[\underline{3}$.

In the same way, we now analyse the scaling of the thermoremanent magnetization. We stress that no use is made of eq. (6) at this point. Introducing the same splitting of the integral, we find from the definition (7) the scaling form $M_{\mathrm{TRM}}(t, s) / h=\rho_{0}+s^{-a} f_{M}(t / s)+$ $s^{-\lambda_{R} / z} g_{M}(t / s)$ where we used the asymptotic forms of $R$ of the three regimes. Then $f_{M}(x)$ is related to $f_{R}(x)[9]$ and the last term (and consequently $g_{M}(x)$, see eq. (8)) was roughly estimated from the mean-value theorem. The term $\rho_{0}$ is related to the initial state of the system. In particular, $\rho_{0}=0$ for an initially fully disordered state. While this expression for $M_{\text {TRM }}$ depends only on the scaling (2), from local scale invariance as introduced and tested in 9, 10, we have eq. (4) and recover (8).

We are now ready for numerical tests of (7) and (8). We begin with the kinetic spherical model, formulated in terms of real spin variables $S_{\boldsymbol{x}}(t)$ subject to the (mean) spherical constraint $\sum_{\boldsymbol{x}}\left\langle S_{\boldsymbol{x}}(t)^{2}\right\rangle=\mathcal{N}$ where $\mathcal{N}$ is the number of sites (see 23] for a careful discussion on this point). The kinetics is given through a Langevin equation

$$
\frac{\mathrm{d} S_{\boldsymbol{x}}(t)}{\mathrm{d} t}=\sum_{\boldsymbol{y}(\boldsymbol{x})} S_{\boldsymbol{y}}(t)-(2 d+\mathfrak{z}(t)) S_{\boldsymbol{x}}(t)+\eta_{\boldsymbol{x}}(t)
$$

where the sum over $\boldsymbol{y}$ extends over the nearest neighbours of $\boldsymbol{x}$. The thermal noise is assumed to satisfy $\left\langle\eta_{\boldsymbol{x}}(t) \eta_{\boldsymbol{y}}\left(t^{\prime}\right)\right\rangle=2 T \delta_{\boldsymbol{x}, \boldsymbol{y}} \delta\left(t-t^{\prime}\right)$. From the spherical constraint the Lagrange multiplier $\mathfrak{z}(t)$ can be found through the solution of a Volterra integral equation, and two-time correlators and response functions are readily found, see [14, 16, 24, for details. 

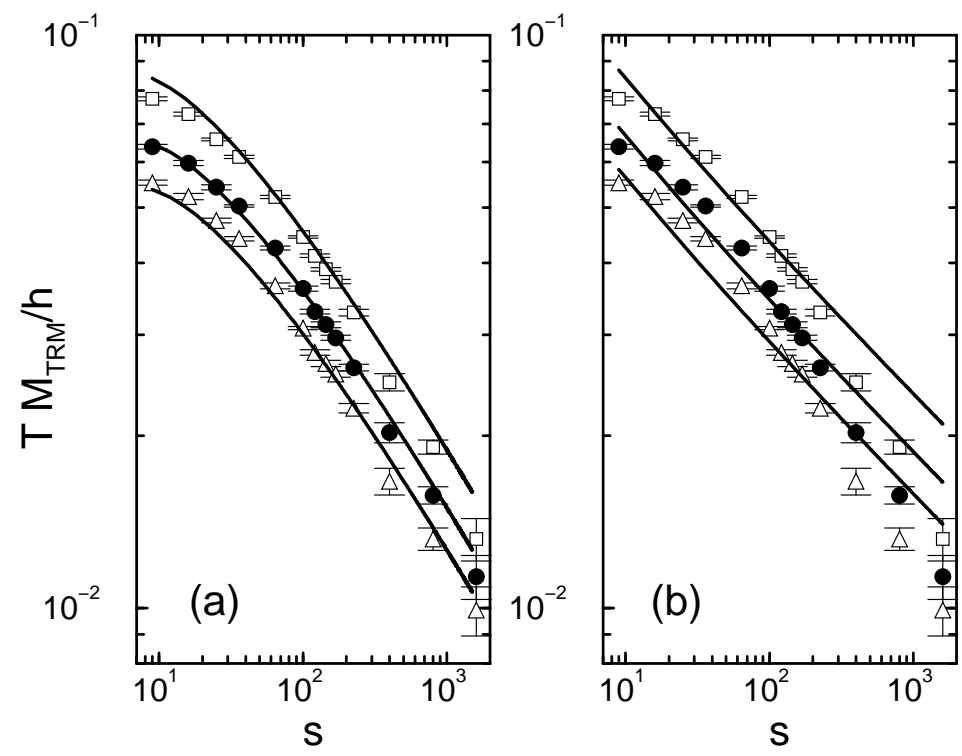

Fig. 2 - Scaling of the thermoremanent magnetization $M_{\mathrm{TRM}}(t, s)$ as a function of $s$ for several values of $x=t / s$ (squares: $x=5$, circles: $x=7$, triangles: $x=9$ ), as obtained in the $2 D$ Glauber-Ising model at $T=1.5$. The black curves are calculated from equations (7) and (8) with $\lambda_{R}=1.26$, see [5] 10]; (a) $a=1 / 2$ and (b) $a=1 / 4$.

In figure \we show the thermoremanent magnetization $M_{\text {TRM }}$ obtained from numerically integrating the Langevin equation, at a temperature $T<T_{c}$ (recall that for $d=3, T_{c} \simeq 3.96$ and for $\left.d=3.5, T_{c} \simeq 5.27\right)$. As an initial state, we used uncorrelated spins with either a mean magnetization $S_{0}=0$ (figure 1 ) or else $S_{0}=0.5$ (figure 1b and 1 1 ). Then the exponents $z=2, a=d / 2-1$ and $\lambda_{R}=d / 2$ for $S_{0}=0$ or $\lambda_{R}=d$ for $S_{0}=0.5$, respectively, are expected [7]. The leading term $\sim s^{-a}$ and the sum of the two leading contributions to $M_{\mathrm{TRM}}$ according to eq. (7) are shown and are compared with the numerical solution of the Langevin equation 25]. For $S_{0}=0.5$, the cross-over between the two regimes is evident. On the other hand, for $S_{0}=0$, we observe that although the slope of $\ln M_{\mathrm{TRM}}$ versus $\ln s$ appears to be fairly constant, the second term expected from eq. (7) produces a sizable correction. We shall find a similar behaviour for the $2 D$ Glauber-Ising model below. Finally, we see that down to values of $s$ as small as $s \sim 1$, the time-dependence of $M_{\text {TRM }}$ is well described by eq. (7). We observed a similar behaviour, and in quantitative agreement with the scaling functions (8), for other values of $x$.

We now come to our main objective: deciding between $a=1 / 2$ and $a=1 / 4$ through a test of (7) in the $2 D$ Glauber-Ising model. This model describes the evolution of the spin variables $s_{j}= \pm 1$ on the sites $j$ of a hypercubic lattice, realized through heat-bath Glauber dynamics. We use a temperature $T=1.5<T_{c}$ and a spatially random field $h= \pm 0.05$ and measure $M_{\mathrm{TRM}}(t, s)$ in the standard way 26 . Starting from a totally disordered state, we measure the evolution of $M_{\mathrm{TRM}}(t, s)$ as a function of $s$ with $x=t / s$ fixed. The non-universal parameters $r_{0,1}$ were fixed using the data for $x=7$ for either $a=1 / 2\left(r_{0}=1.76, r_{1}=-1.84\right)$ or $a=1 / 4\left(r_{0}=0.22, r_{1}=0.09\right)$, respectively. Since $r_{0,1}$ are independent of $x$, we therefore obtain a prediction on how $M_{\mathrm{TRM}}(x s, s)$ should scale for any other value of $x$. This is shown in figure 2] While for $a=1 / 2$, the Monte Carlo data nicely agree with the cross-over scaling as 
predicted from eqs. (78), it is also clear from figure 2]b that the hypothesis $a=1 / 4$ [18, 19] is incompatible with the data. The same conclusion, namely the invalidity of the value $a=1 / 4$ in the $2 D$ Glauber-Ising model, has also been reached using simulational data at $T=0$ with waiting times up to $s=5600$ [27].

Summarizing, we have reanalysed the scaling of the magnetic linear response in simple ferromagnets undergoing phase-ordering kinetics. The cross-over scaling of the thermoremanent magnetiztion eq. (7) (and also the dynamic susceptibility) is predicted exactly for any dynamic universality class from local scale invariance in terms of the universal exponents $\lambda_{R} / z$ and $a$. We have confirmed eqs. (6) and (78) in the Glauber-Ising and spherical models. This provides evidence against recent suggestions of an anomalous scaling of the dynamic suceptibilities in phase-ordering kinetics.

M. Paeßens is grateful to the Deutscher Akademischer Austauschdienst (DAAD) for financial support. This work was supported by the Bayerisch-Französisches Hochschulzentrum (BFHZ) and by CINES Montpellier (projet pmn2095).

\section{REFERENCES}

[1] J.P. Bouchaud in M.E. Cates and M.R. Evans (eds), Soft and Fragile Matter, IOP (Bristol 2000).

[2] A.J. Bray, Adv. Phys. 43, 357 (1994).

[3] C. Godrèche and J.M. Luck, J. Phys. Cond. Matt. 14, 1589 (2002).

[4] L.F. Cugliandolo, cond-mat/0210312

[5] D.S. Fisher and D.A. Huse, Phys. Rev. B38, 373 (1988).

[6] T.J. Newman and A.J. Bray, J. Phys. A23, 4491 (1990).

[7] A. Picone and M. Henkel, J. Phys A35, 5575 (2002).

[8] C. Yeung, M. Rao and R.C. Desai, Phys. Rev. E53, 3073 (1996).

[9] M. Henkel, Nucl. Phys. B641, 405 (2002).

[10] M. Henkel, M. Pleimling, C. Godrèche and J.-M. Luck, Phys. Rev. Lett. 87, 265701 (2001).

[11] C. Godrèche and J.M. Luck, J. Phys. A33, 1151 (2000).

[12] E. Lippiello and M. Zannetti, Phys. Rev. E61, 3369 (2000).

[13] L. Berthier, J.L. Barrat, and J. Kurchan, Eur. Phys. J. B11, 635 (1999).

[14] L.F. Cugliandolo and D.S. Dean, J. Phys. A28, 4213 (1995).

[15] W. Zippold, R. Kühn and H. Horner, Eur. Phys. J. B13, 531 (2000).

[16] C. Godrèche and J.M. Luck, J. Phys. A33, 9141 (2000).

[17] F. Corberi, E. Lippiello and M. Zannetti, Eur. Phys. J. B24, 359 (2001).

[18] F. Corberi, E. Lippiello and M. Zannetti, Phys. Rev. E65, 046136 (2002).

[19] F. Corberi, E. Lippiello and M. Zannetti, Phys. Rev. Lett. 90, 099601 (2003).

[20] The kinetics of the $1 D$ Glauber-Ising model is very peculiar since its critical temperature is $T_{c}=0$. It appears to fit into class $\mathrm{L}$ rather than class $\mathrm{S}$.

[21] L. Berthier, P.C.W. Holdsworth, and M. Sellitto, J. Phys. A34, 1805 (2001).

[22] S.A. Cannas, D.A. Stariolo and F.A. Tamarit, Physica A294, 362 (2001).

[23] N. Fusco and M. Zannetti, Phys. Rev. E66, 066113 (2002).

[24] M. Paessens and M. Henkel, to be published.

[25] In figure 1 we have (a) $r_{0} \simeq 0.057, r_{1} \simeq-0.0235$, (b) $r_{0} \simeq 0.0047, r_{1} \simeq 0.00061$, (c) $r_{0} \simeq$ $0.0017, r_{1} \simeq 0.00028$.

[26] A. Barrat, Phys. Rev. E57, 3629 (1998).

[27] M. Henkel and M. Pleimling, Phys. Rev. Lett. 90, 099602 (2003). 